\title{
Systemic Lupus Erythematosus Patient with Bicytopenia
}

\section{and Motor Aphasia}

\author{
Mira Delima Asikin ${ }^{\mathrm{a}}$, Awalia $^{\mathrm{b} *}$ \\ a mirarsuntan@gmail.com \\ ${ }^{a}$ Resident, Internal Medicine Department Faculty of Medicine Universitas Airlangga, Dr. Soetomo Teaching Hospital Jl. Prof. dr. \\ Moestopo 6-8, Surabaya 60286, Indonesia \\ ${ }^{b}$ Internist, Division of Rheumatology Internal Medicine Department Faculty of Medicine Universitas Airlangga, Dr. Soetomo Teaching \\ Hospital Jl. Prof. dr. Moestopo 6-8, Surabaya 60286, Indonesia
}

\begin{abstract}
Background: Systemic lupus erythematosus (SLE) is a chronic inflammatory autoimmune condition with unclear cause that shows a broad ranging clinical symptoms, course and prognosis. Cerebrovascular illness, a severe symptom SLE in the central nervous system by neuropsychiatric SLE (NPSLE). Aphasia is considered to be one of the rarer signs, produced by secondary antiphospolipid syndrome (APS), of a cerebrovascular condition of NPSLE.

Case: A 37 years-old married woman that has background of miscarriage in SLE with speech blocking, motor aphasia, and had nasal voice was diagnosed with NPSLE with APS. After initiating therapy with cyclophospamide and aspilet the patient's clinical condition improved significantly.

Conclusion: APS needs to bear in mind in SLE patients that has background of miscarriage or thrombosis. The diagnosis was based on history, clinical, and laboratory examinations which include phospholipid antibodies, There was dramatic improvement after 12 days of $500 \mathrm{mg}$ of cyclophospamide and oral aspilet $100 \mathrm{mg}$ daily therapy.
\end{abstract}

Keywords: Neuropychiatric systemic lupus erythematosus; antiphospolipid syndrome; aphasia; bicytopenia

\section{Introduction}

Systemic lupus erythematosus (SLE) is a chronic inflammatory autoimmune condition with unclear cause that shows a broad ranging clinical symptoms, course and prognosis. The disease mostly happens to women with such a significant mortality rate in reproductive age. The World Health Organization (WHO) noted that the number of people with lupus globally has reached five million people. The incidence is 5.1 per 100,000 people in the United States, the proported women to men ranges from 9:1 to 14:1. In Indonesia, the total number of people with lupus is still unclear. The percentage of SLE patients in the community according to a survey conducted by Prof. Handono Kalim, et al in Malang was approximately $0.5 \%$ of the total 
population (Perhimpunan Rheumatologi Indonesia, 2011; Pusdatin, 2017).

The clinical manifestations of SLE are variable, which includes involvements of skin and mucosa, joints, blood, heart, lungs, kidneys, central nervous system (CNS), and immune system. Bicytopenia is a decrease of two blood components, which could be in the form of anemia, thrombocytopenia, and leukopenia. The manifestation of blood disorders in SLE could be in the form of all three or two of them, and these three blood components could determine the severity of SLE (Perhimpunan Rheumatologi Indonesia, 2011; Durrani et al., 2015).

Neuropsychiatric involvement could be present in SLE, which is named as neuropsychiatric SLE (NPSLE). NPSLE often occurs in $39-50 \%$ of patients with SLE, one of which is cerebrovascular disease, a serious complication of SLE in the central nervous system occurring in $30.76 \%$ of patients. This manifestation could reduce quality of life, increase morbidity and mortality of SLE, and has a poor prognosis. (Checa et al., 2016; Kakati et al., 2017).

Motor aphasia is a deficiency in speech production or understanding. Cerebrovascular disorder, particularly cerebral infarction, responsible for between $15-38 \%$ of ischemic stroke, is the major cause of aphasia. Aphasia could also be caused by other diseases such as trauma, tumors, degenerative diseases, and metabolic diseases (Fontanesi \& Schmidt, 2016; Clark, 2017).

Aphasia in this patient is thought to be one of the rare manifestations of cerebrovascular disease from NPSLE, which could be caused by secondary antiphospolipid syndromes and requires complicated therapy (Bertsias et al., 2012). Therefore, we present this case.

\section{Case Presentation}

A married female, 37 years of age, Betawi, came to the ER with a vaginal bleeding complaint that had been worsened during the last two days outside her monthly cycle since a week ago. The patient had to change her menstrual pads for six time in a day. She was hospitalized in ward with a diagnosis of abnormal uterine bleeding. In addition, the patient also complained of dizziness, weakness, fatigue, lethargy, blurry vision and difficulty speaking. The patient had experienced hair loss since she was young and joint pain in the last 5 years. She denied any rash on the face nor mouth sores. The patient previously visited gynecology emergency unit of Dr. Soetomo General Hospital and was diagnosed with a cyst and was given medication, where her bleeding managed to stop and the patient has been released from the hospital. The patient said she had several years ago a history of lupus and had never taken any medicines. She first became pregnant in 2011 and suffered a 2-month gestation miscarriage, her second pregnancy was in 2012 and her baby was born prematurely at 7 months of gestation and did not survive, her third pregnancy was in 2013 and her baby was 
born prematurely by caesarean section at 7 months of gestation and the child survived until present day.

Physical examination revealed weak general condition with full awareness, but she experienced speech blocking, difficulty speaking (motor aphasia) and had nasal voice (but still able to understand speech), blood pressure of 100/70 $\mathrm{mmHg}$, heart rate of $84 \mathrm{x} /$ minute, respiratory rate of $18 \mathrm{x} /$ minute, and axillary temperature of $36^{\circ} \mathrm{C}$. Head and neck examinations revealed anemic conjunctiva and no rash was found. Chest and abdominal examinations revealed no abnormality was found. Extremities examination revealed warm, dry, and pale acral with petechiae in the lower extremities.

Laboratory tests to evaluate the patient's bleeding revealed no abnormalities, except for a decrease in hemoglobin (Hb) (5.4 g/dl), MCV (74), MCHC (24), lymphocytes (9\%) and platelets (16,000). The patient's pregnancy urine test was negative and gynecological ultrasound showed no abnormalities. From these data, the patient was diagnosed with microcytic hypochromic anemia, suspected SLE-related thrombocytopenia with differential diagnosis of idiopathic purpura thrombocytopenia and antiphospolipid syndrome. The patient was given $2100 \mathrm{kcal}$ high protein high calorie diet per day, a pulse dose of $500 \mathrm{mg}$ methyprednisolone injection for 3 days followed by $1 \mathrm{mg} / \mathrm{kgBW} /$ day, 2 units of PRC transfusion/day and 10 units of Thrombocyte (TC) transfusion/day.

After 5 days of treatment, her bleeding stopped. The patient general condition was fair, but the patient still had difficulty speaking, nasal sound, and speech blocking. Peripheral blood revealed that her $\mathrm{Hb}$ and lymphocyte counts were still the similar to previous results, but her platelet count increased to 234,000 . ANA test was positive 115.78, while her C3, C4, and CRP were normal. The patient was referred for an urgent head CT scan without contrast, and the result was subacute ischemic cerebral infarction on the left corona radiata. At that time, there was difficulty to find suitable blood for transfusion because the results of the patient's red blood cell examination revealed invivo sensitization by anti-IgG with positive $\mathrm{C} 3 \mathrm{~d}$ complement factor and auto control 4. The patient was diagnosed with NPSLE and suspected secondary antiphospolipid syndrome. The patient was then given 2 units of wash erythrocyte (WE) transfusion/day, and cyclophospamide $500 \mathrm{mg}$ was planned if the patient's $\mathrm{Hb}$ target was met.

After 10 days of treatment, the patient's condition was still similar to her previous condition. Peripheral blood examination revealed an increased of $\mathrm{Hb}$ to 10.5 with a platelet count of 88,000 without significant bleeding after WE transfusion. The patient was then tested for IgG and IgM ACA with negative IgG result and positive IgM result. A lupus anticoagulant examination also revealed positive result. According to antiphospholipid syndrome diagnostic criteria, if there is one clinical criteria, in this case thrombosis through imaging, and one laboratory criteria, in this case positive LA, the patient could be diagnosed with secondary antiphospholipid syndrome with SLE. The patient was given $500 \mathrm{mg}$ of cyclophospamide therapy and 
received additional 1x100 mg of aspilet therapy.

The patients were able to speak smoothly after 12 days of treatment, and their blockage of speech eased progressively. The patient was then discharged from the hospital and continued her treatment in the outpatient clinic.

\section{Discussion}

SLE is an autoimmune chronic illness that could affect practically all organ systems. Clinical manifestations could be found in the form of acute cutaneous lesions such as malar rash, discoid rash, photosensitivity, arthritis, serositis, kidney, neurological, and haematological disorders. Hematological disorder that is most commonly found in SLE patients is autoimmune hemolytic anemia, which often coexist with thrombocytopenia and lymphopenia (Domiciano \& Shinjo, 2010; Perhimpunan Rheumatologi Indonesia, 2011; Kuhn et al., 2015).

Spontaneous bleeding manifestation often occurs in severe thrombocytopenia with a platelet count of $<30,000 /$ microLiter. Spontaneous bleeding that might occur is in the form of petechiae, gingival, gastrointestinal, and genitourinary bleeding (Gauer \& Braun, 2012; Goerge \& Arnold, 2018).

In this patient, there was vaginal bleeding outside her menstrual cycle since 1 week ago, worsen in the last two days, and the patient must changed her menstrual pads 6 times per day, with a diagnosis of abnormal uterine bleeding and was treated in obstetrics and gynecology ward. The patient's laboratory examination revealed anemia with auto-control examination result of +4 and was suspected with autoimmune hemolytic anemia (Hb $5.4 \mathrm{~g} / \mathrm{dL}$ ) and thrombocytopenia (platelet count of 16,000/microLiter).

The diagnostic criteria for SLE are as follows, According to the 1997 amend by American College of Rheumatology (ACR): malar rash, discoid rash, photosensitivity, mouth ulcers, arthritis, serositis (pleurisy or pericarditis), renal disorders, neurological disorders, haematological disorders (hemolytic anemia) with reticulosis, or leucopenia fewer than $4,000 / \mathrm{mm}^{3}$, or lymphopenia fewer than $1,500 / \mathrm{mm}^{3}$, or thrombocytopenia fewer than $100,000 / \mathrm{mm}^{3}$ without being caused by drugs, and immunologic disorders (positive ANA/ antinuclear antibody). If 4 or more of these criteria are found, SLE diagnosis has a $85 \%$ sensitivity and a $95 \%$ specificity \%. Meanwhile, if there are only 3 criteria are met and one of them is ANA positive, SLE diagnosis is highly possible and the diagnosis will depend on clinical observation. If ANA test is negative, other diagnosis must be thought besides SLE. If there is only a positive ANA test and other clinical manifestations are not present, it might not be SLE and long-term observation is needed (Perhimpunan Rheumatologi Indonesia, 2011).

This patient experienced joint paint in the last 5 years. Hemolytic anemia, thrombocytopenia, and 
positive ANA test were present. According to the above SLE criteria, this patient met 3 diagnosis criteria and one of them is positive ANA test, which indicated a high possibility of SLE.

Neuropsychiatric SLE(NPSLE) is the closely associated neurological and psychiatric symptoms of SLE. The clinical manifestations of NPSLE could be classified as central (12 manifestations) or peripheral (7 manifestations). The central manifestations are divided into 8 neurological syndromes (aseptic meningitis, cerebrovascular disease, demyelination syndrome, headache, movement disorders, myelopathy, seizures, acute confusional state) and 4 psychiatric syndromes (anxiety disorders, cognitive dysfunction, mood disorders, psychosis). Peripheral manifestations include Guillain-Barre' syndrome, autonomic disorders, mononeuropathy, myasthenia gravis, cranial neuropathy, plexopathy, polyneuropathy. Several research show that cognitive impairment and headaches are the most prevalent signs (Perhimpunan Rheumatologi Indonesia, 2011; Checa et al., 2016).

Broad manifestations of NPSLE ranges from central to peripheral nerve dysfunction, as mentioned above, and from mild cognitive symptoms to severe neurological manifestations such as stroke (Perhimpunan Rheumatologi Indonesia, 2011). There are two hypotheses that might explain NPSLE pathophysiology (Checa et al., 2016). Autoimmune or inflammatory mechanisms caused by autoantibodies or inflammatory mediators that induces brain dysfunction and interfere with blood-brain barrier or intrathecal formation of immune complexes and vascular damage and occlusion, resulting from thrombotic processes in both small and large intracanal blood vessels due to autoantibodies mediated by vascular injury, immune complexes, complement deposition, leukoagglutination, and atherosclerosis.

On physical examination, her general condition was weak with full awareness, but speech blocking and difficulty speaking (motor aphasia) were present as well as nasal sound. The patient was referred to urgent head CT scan without contrast, and the result was subacute ischemic cerebral infarction on the left corona radiata. Motor aphasia is a deficiency in speech production or understanding. Cerebrovascular disorder, particularly cerebral infarction, responsible for between $15-38 \%$ of ischemic stroke, is the major cause of aphasia (Clark, 2017).

In analysis, aphasia is classified into two types: sensory and motor. Sensory aphasia is a loss in understanding language, while motor aphasia is a loss of ability in articulation, fluency, repetition, and naming (Gupta \& Singhal, 2011). The patient had motor aphasia in the form of speech difficulties and blocking, but the patient could understand speech. Aphasia in SLE patients could be one of the manifestations of cerebrovascular disease in Neuropsychiatry SLE (NPSLE).

The patient had cerebral infarction followed by severe thrombocytopenia. The patient also has background of miscarriage from her first pregnancy in 2011, and of premature labor during her second 
pregnancy, in 2012, at seven months pregnant, where her baby did not survive, and another premature labor via caesarean section in 2013. Antiphospolipid syndrome (APS) needs to be considered in SLE patients with history of miscarriage or thrombosis. From history taking and further examinations, the results highly suggested the presence of antipohospolipid syndrome (APS). Thrombocytopenia is known to be an early complication in patients with lupus anticoagulant activity (Cuadrado et al., 1997). Afterwards, the patient was tested for IgG ACA (anticardiolipin) with a negative result, while her IgM ACA revealed a positive result. The patient was then tested with lupus anticoagulant (LA) test, which also revealed a positive result.

APS is a systemic autoimmune disorder distinguished as a result of arterial or venous thrombosis and/or pregnancy, including repeated miscarriage by the existence of phospholipid antibody (aPL) in healthy women. APS might occur primarily or in secondary to SLE or other immune system diseases (Puerta \& Cervera, 2013; Erkan \& Ortel, 2017). In this patient, APS was secondary to SLE. aPL is a heterogeneous group of antibodies that directly attacks phospholipid-binding proteins. Those included in this group are anticardiolipin (ACA) antibodies, either IgG or IgM enzyme-linked immunosorbent assay (ELISA), anti-beta2-glycoprotein (GP) I antibody (IgG or IgM) ELISA, and lupus anticoagulant (LA) assay. (Puerta \& Cervera, 2013; Erkan \& Ortel, 2017).

In the early 1960s, 30 years ago Graham R.V. Hughes identified a link between thrombosis, fetal error and thrombocytopenia with tLA. He combined cerebral ailment (stroke) with abortion and LA. Data available suggests that APS-related autoantibodies directly assault a variety of plasma proteins and endothelial-binding plasma proteins/platelets. In normal anticoagulant and pro-coagulant response and in some cells that might modify the expression and secretion of diverse molecules Phospholipid antibodies involvement is clinical significant. This occurrence might provide as the foundation for aPL in APS patients to trigger thrombotic events. (Puerta \& Cervera, 2013).

Antiphospholipid syndrome classification criteria are as follows clinical criteria and laboratory criteria. Clinical criteria there are two point vascular thrombosis and pregnancy morbidity. Laboratory criteria there are three point : IgG and or IgM cardiolipin antibody (ACA) in serum or plasma with medium or high titers ( $>$ 40) were measured by ELISA method; Positive Lupus anticoagulant in serum, and IgG or IgM anti-B2 glycoprotein (Puerta \& Cervera, 2013; Perhimpunan Reumatologi Indonesia, 2011) A patient is diagnosed with APS if a minimum of one clinical criteria and one laboratory criteria are met with a minimum time of 12 weeks since clinical manifestations. According to Puerta \& Cervera (2013), the ACA parameter has the highest immulogical percentage, which is $87.9 \%$. Therefore, this patient with suspected APS was first tested for ACA, with a negative IgG result and a low positive IgM. To support these findings, another parameter was tested with the second highest immunological percentage (LA), and the result was positive. 
In accordance with SLE management flowchart from Indonesian Rheumatology Association, in initial management, the patient was given $500 \mathrm{mg}$ of methylpredinosolone pulse induction therapy for 3 days, with the addition of $500 \mathrm{mg}$ cyclophosphamide. Cyclophosphamide was indicated because of hematological disorder in the form of severe thrombocytopenia with bleeding and hemolytic anemia as well as NPSLE. These are management for severe SLE. Management of APS is basically aimed for thrombosis incidence management in this patient, where she received a small dose of aspirin $(80 \mathrm{mg} /$ day $)$. Aspirin could be considered for SLE patients with APS as primary prevention against thrombosis and miscarriage (Perhimpunan Rheumatology Indonesia, 2011).

According to Estel et al (2016), the 15-year survival of SLE with APS is worse than SLE without APS (65\% \& 95\%). The presence of APS in SLE has a higher mortality rate. The main cause of death includes stroke, myocardial infarction, and pulmonary embolism.

\section{Conclusion}

It has been reported that a female patient aged 37 years was diagnosed with NPSLE with secondary APS presenting with bicytopenia and motor aphasia. The risk factor for APS in patients was due to SLE. The diagnosis of NPSLE and APS was based on history, clinical, and laboratory examinations which include phospholipid antibodies, in this case IgG ACA, IgM ACA, and LA. The patient's prognosis is poor.

\section{Acknowledgements}

I like to express my appreciation of the unending support and prayer of all the educators at the Division of Rheumatology, the Internal Medicine Department, Faculty of Medicine of the University of Airlangga as well as of my family and friends.

\section{References}

Bertsias G, Cervera R \& Boumpas DT. 2012. Systemic Lupus Erythematosus: Pathogenesis and Clinical Features. Eular Textbook on Rheumatic Disease. Switzerland: BMJ.

Checa CM, Zirkzee EJ, Huizing TW \& Beekman GM, 2016. Management of Neuropsychiatric Systemic Lupus Erythematosus: Current Approaches and Future Perspectives. Springer Open Choice Journal. 76: 459-483.

Clark DG. 2017. Aphasia: Prognosis and Treatment. Available from: https://www.uptodate.com/contents/aphasia-prognosis-andtreatment.

Cuadrado MJ, Mujic F, Munoz E, Khamasta MA \& Hughes GR, 1997. Thrombocytopenia in the antiphospholipid Syndrome. Annals of the Rheumatic Diseases. 56:194-196.

Domiciano DS \& Shinjo SK. 2010. Autoimmune hemolytic anemia in systemic lupus erythematosus: association with thrombocytopenia. Clin Rheumatol Journal. 29(12):1427-31.

Durrani SH, Sayyar M, Lal A \& Aslam R. 2015. Incidentally Diagnosed Bicytopenia Showing A Wide Spectrum of Pathologies on Bone Marrow Morphology. Khyber Journal of Medicine Science. 8: 2.

Erkan D \& Ortel TL. 2017. Diagnosis of antiphospholipid syndrome. Available from: https://www.uptodate.com/contents/diagnosis-ofantiphospholipid-syndrome. 
Estel GJ, Andreoli L, Scanzi F, Cervera R \& Tincani A. 2016. The Antiphospholipid Syndrome in Patients with Systemic Lupus Erythematosus. Journal of Autoimmunity. 1-11.

Fontanesi SR \& Schmidt A. 2016. Interventions in aphasia: an integrative review. Speech, Language, Hearing Sciences and Education Journal. 18(1):252-262.

Gauer RL \& Braun MM. 2012. Thrombocytopenia. Am Fam Physician Journal. 85(6):612-622.

Goerge JN \& Arnold DM. 2018. Approach to the adult with unexplained thrombocytopenia. Available from: https://www.uptodate.com/contents/approach-to-the-adult-with-unexplained-thrombocytopenia.

Gupta A \& Singhal G. 2011. Understanding Aphasia in a Simplified Manner. Journal Indian Academy of Clinical Medicine. 12(1): 32-7.

Kakati S, Barman B, Ahmed SU \& Hussain M, 2017. Neurological Manifestations in Systemic Lupus Erythematosus: A Single Centre Study from North East India. Journal of Clinical and Diagnostic Research. 11(1): OC05-OC09.

Kuhn A, Bonsmann G, Anders HJ, Herzer P, Tenbrock K \& Schneider M. 2015. The Diagnosis and Treatment of Systemic Lupus Erythematosus. Deutsches Ärzteblatt International Dtsch Arztebl Int. 112: 423-32.

Perhimpunan Rheumatologi Indonesia. 2011. Diagnosis dan Pengelolaan Lupus Eritematosus Sistemik. Jakarta: Perhimpunan Rheumatologi Indonesia.

Puerta \& Cervera, 2013. Diagnosis and Classification of the Antiphospolipid Syndrome. Journal of Autoimmunity. 48-49: 20-25.

Pusdatin, 2017. Situasi Lupus di Indonesia. Jakarta: Pusdatin. 